\title{
«smarter medicine»: la liste «Top-5» de la SRO
}

\section{Organisation responsable «smarter medicine»}

La Société américaine de radio-oncologie (ASTRO) avait publié, en 2013 et 2014, une liste top-5 des traitements radio-oncologiques spécifiques qui, tout en étant fréquemment réalisés, ne semblent toutefois ni efficaces ni adéquats dans les circonstances données. Ces discussions ont été suivies avec grand intérêt par la Société suisse de radio-oncologie (SRO) qui a ensuite chargé son comité de direction de l'évaluation et de l'élaboration d'une telle liste top-5 pour le domaine de la radio-oncologie en Suisse.

La SRO souhaite amorcer une discussion sur le thème de la prise en charge radio-oncologique conforme aux besoins des patientes et des patients souffrant d'un cancer, non seulement au sein des sociétés de discipline, mais également au niveau multidisciplinaire et interprofessionnel, pour le bien des personnes concernées et de leurs proches, ainsi que pour les professionnels de la santé impliqués.

La Société suisse de radio-oncologie recommande de ne pas pratiquer les interventions suivantes en radiooncologie:

1. Ne pas démarrer de radiothérapie du sein entier dans le cadre d'un traitement conservateur du sein chez les femmes de $\mathbf{5 0}$ ans et plus à un stade précoce de cancer du sein invasif, sans prendre en compte des programmes de traitement plus courts.

- La radiothérapie du sein entier diminue la récurrence locale et améliore le pronostic de survie des femmes atteintes de cancer du sein invasif et ayant bénéficié d'un traitement conservateur du sein. La plupart des études ont utilisé des programmes «conventionnellement fractionnés» comportant une thérapie de cinq à six semaines, souvent suivie d'une irradiation additionnelle (boost) d'une à deux semaines.

- Des études récentes ont néanmoins démontré un contrôle de la tumeur et un résultat cosmétique équivalents parmi des populations spécifiques de patientes ayant subi des traitements plus courts (environ quatre semaines). Il appartient aux patientes et à leurs médecins de passer en revue de telles options pour déterminer le traitement le plus adapté.

\section{La campagne "smarter medicine»}

Une nouvelle association de soutien dynamise en Suisse I'initiative "smarter medicine» lancée en 2014. Les orientations de la campagne sont soutenues par des organisations médicales spécialisées et professionnelles, mais aussi par des associations qui défendent les intérêts des patients et des consommateurs. Ensemble, elles souhaitent sensibiliser le public au fait qu'en médecine et pour certains traitements, moins peut parfois signifier plus de qualité de vie pour les personnes concernées.

"Smarter medicine» s'est adaptée à l'initiative américaine "Choosing Wisely" qui remporte un grand succès et qui a pour but non seulement de déboucher sur des «décisions judicieuses", mais aussi d'encourager une franche discussion entre le corps médical, les patients, et le public.

Dans les prochains mois, d'autres sociétés de disciplines publieront leurs listes Top- 5 avec des recommandations pour leurs domaines d'activité

Vous trouverez plus d'informations concernant l'asscociation de soutien et les listes Top-5 publiées sous www.smartermedicine.ch.

2. Ne pas commencer la prise en charge d'un cancer de la prostate à faible risque sans avoir envisagé une surveillance active.

- Les patients atteints d'un cancer de la prostate disposent de plusieurs options raisonnables de prise en charge. Celles-ci comprennent la chirurgie et la radiothérapie, mais aussi la surveillance conservatrice sans traitement lorsque le cas du patient s'y prête.

- Le partage de la prise de décision entre le patient et son médecin peut permettre de mieux faire correspondre le projet de soins du patient et le traitement, et de garantir davantage d'efficacité dans la dispense des soins.

- L'ASTRO a publié des documents d'aide à la décision destinés aux patients concernant le cancer de la prostate et de nombreux autres types de cancers. Ces types d'instruments peuvent aider les patients à faire leurs choix avec davantage d'assurance pour être en meilleure adéquation avec leur traitement.

3. Ne pas utiliser systématiquement des programmes de fractionnement prolongés (plus de dix séances) dans le traitement palliatif des métastases osseuses. 


\section{Élaboration de cette liste}

Après I'adhésion de I'American Society for Radiation Oncology (ASTRO) à la campagne Choosing Wisely, une enquête a été réalisée auprès des comités Health Policy, Government Relations, et Clinical Affairs and Quality de I'ASTRO, afin d'identifier les éléments potentiels à inclure dans la liste. Par ailleurs, un groupe de travail composé de médecins exerçant dans ces domaines a été instauré et convoqué. Ses membres ont été priés de définir leurs préférences parmi les thèmes proposés dans l'enquête initiale. En janvier 2014, I'ASTRO a constitué un groupe de travail chargé d'élaborer une seconde liste Choosing Wisely; celui-ci comprend également des représentants des comités Health Policy, Government Relations, et Clinical Affairs and Quality. Dans un premier temps, le groupe de travail a raccourci la liste des propositions. Une enquête électronique anonyme a ensuite été envoyée aux membres de l'ASTRO pour évaluer la valeur et la pertinence de chacun des éléments. Le conseil d'administration de I'ASTRO $s^{\prime}$ est chargé de la sélection définitive des éléments à soumettre. La Société suisse de radio-oncologie s'est appuyée sur la liste "Choosing Wisley" publiée par I'American Society for Radiation Oncology et a jugé une sélection de recommandations applicable et pertinente pour la Suisse.

Une liste détaillée des références et des recommandations d'autres sociétés médicales sont disponibles en ligne sous www. smartermedicine.ch.

- Les études suggèrent un soulagement de la douleur équivalent après $30 \mathrm{~Gy}$ administrés sur dix séances, 20 Gy sur cinq séances, ou 8 Gy en une séance unique.

- Une séance unique est moins contraignante mais peut impliquer un taux plus élevé de retraitement sur la même zone.

- Une séance unique de 8 Gy doit être envisagée en priorité pour les patients au pronostic de survie limité ou ayant des difficultés de transport.

4. Ne pas conseiller de radiothérapie à la suite d'une hystérectomie pour les patientes atteintes de cancer de l'endomètre à faible risque.

- Les patientes atteintes de cancer de l'endomètre à faible risque (y compris celles ne présentant plus de cellules malades à l'issue d'une hystérectomie, malgré une biopsie positive), présentant une extension tumorale de grade 1 ou 2, une invasion myométriale inférieure à 50\% et sans caractéristiques supplémentaires à haut risque telles que l'âge (plus de 60 ans), une invasion de l'espace lymphovasculaire ou une implication cervicale, présentent un risque de récurrence très faible à l'issue de l'intervention chirurgicale.

- Les études de méta-analyse de la radiothérapie pour le cancer de l'endomètre à faible risque témoignent d'effets secondaires accrus sans amélioration du pronostic de survie global par rapport à la chirurgie seule.

5. Ne pas ajouter systématiquement de radiothérapie cérébrale totale à la radiochirurgie stéréotaxique pour des métastases cérébrales limitées.

- Des études randomisées n'ont pas montré d'avantage en termes de pronostic de survie global du fait de l'adjonction d'une radiothérapie cérébrale totale à la radiochirurgie stéréotaxique (RCS) dans la prise en charge de patients sélectionnés présentant un bon état physique et des métastases cérébrales de tumeurs solides.

- L'adjonction de la radiothérapie cérébrale complète à la radiochirurgie stéréotaxique entraîne une diminution des fonctions cognitives et, selon les patients, une aggravation de la fatigue et une détérioration de la qualité de vie. Ces résultats corroborent les fonctions cognitives détériorées et les compétences verbales diminuées dont les patients font état dans les études randomisées d'irradiation crânienne prophylactique pour le cancer du poumon à petites cellules ou non à petites cellules.

- Les patients traités par radiochirurgie pour des métastases cérébrales peuvent développer des métastases dans d'autres régions du cerveau. Une surveillance attentive et l'usage judicieux d'un traitement de rattrapage en cas de récidive cérébrale permettent aux patients concernés de profiter d'une excellente qualité de vie sans détérioration du pronostic de survie global. Il appartient aux patients de discuter de ces options avec leur radio-oncologue. 